\title{
Past Present Future of Covid-19 Pandemic Problem in the World
}

\section{Narendra Kumar Chopra ${ }^{1 *}$, Mohammad Saiful Hoq Ibna Majib ${ }^{2}$ and Dassanayake Mudiyanselage ${ }^{3}$}

${ }^{1}$ Clinical Professor, Faculty of Medicine, SEGI University, Malaysia

${ }^{2}$ MBBS. PhD Radiology Senior lecturer and Radiologist, Malaysia

${ }^{3} \mathrm{MBBS}$, Malaysia

*Corresponding Author: Narendra Kumar Chopra, Clinical Professor, Faculty of

Medicine, SEGI University, Malaysia.
Received: March 10, 2021

Published: March 22, 2021

C All rights are reserved by Narendra Kumar

Chopra.

\section{Abstract}

The COVID-19 pandemic in its rampage sparked questions in healthcare, administration, economics, community welfare, racial prejudice and politics. Many of the initial cases are believed to be from a common source, the Hunan Seafood Market. China notified the World Health Organization on the $3^{\text {rd }}$ of January 2020 when 27 cases were recognized. Since then it has crossed borders all over the world mainly due to movement during the Lunar New Year Holiday. One week since the notification to WHO, 2019-nCoV was identified.

COVID-19 is caused by SARS CoV-2 with a fatality rate lying approximately between $0.5 \%$ to $10 \%$ worldwide [32] (Figure 5). Patients with underlying systemic illness and the elderly have the highest risk of contracting the disease and having a fatal reaction to it. Human to human transmission via aerosolized respiratory droplets is the most common form of transmission. The majority of all patients $(80 \%)$ are asymptomatic or have a milder presentation. There is insufficient evidence to suggest transmission from asymptomatic patients [33,34]. Early identification, isolation, and symptomatic management of infected patients is the minimum recommended practice worldwide. The use of adjunctive therapy such as hydroxychloroquine and antivirals are not recommended. 6 vaccines are currently entering into the final phase 3 of their clinical trials. Prevention strategies are implemented worldwide to halt the spread of the virus. Masks, movement restrictions, physical distancing, and quarantine measures are a few among the effective. The overview of covid-19 is carried out with the following objectives: We have to live with this pandemic for several years with all precautions of infection control in spite of COVID vaccination which will take several years for development of herd immunity more than 80 percent at international level which will result in decline of new case of COVID-19.

Keywords: COVID-19; ARDS; SARS- COV; MERS-COV; Preventive Interventions; WHO; CDC

\section{Introduction}

COVID-19 is unquestionably the most debilitating pandemic of the $21^{\text {st }}$ century. Caused by a coronavirus virulent enough to infiltrate and infect all the continents, except for Antarctica within a matter of weeks. The pandemic in its rampage sparked questions in healthcare, administration, economics, community welfare, racial prejudice, and politics. Many of the initial cases are believed to be from a common source, the Hunan Seafood Market. China notified the World Health Organization on the $3^{\text {rd }}$ of January 2020 when 27 cases were recognized. Since then, it has crossed borders all over the world mainly due to movement during the Lunar New Year Holiday. One week since the notification to WHO, 2019-nCoV was identified. When comparing COVID-19 to Severe Acute Respiratory Syndrome (SARS) in 2002 and Middle East respiratory syn- 
drome in 2012, there are similarities and differences. SARS affected 8096 cases with 774 deaths within 29 countries. MERS is still uncontained with 2494 confirmed cases and 858 deaths within 27 countries. All 3 outbreaks are caused by a novel coronavirus that crossed over to humans via a zoonotic transmission [1]. On the $30^{\text {th }}$ of January 2020, WHO declared the COVID-19 outbreak a global health emergency and officially named it a pandemic on the $11^{\text {th }}$ of March 2020. To contain the spread, measures such as lockdowns, movement restriction orders, air and transport limitations, work from home and school closures have been implemented to varying extents worldwide. Furthermore, the virus has challenged the limits of healthcare systems in many countries due to the massive influx of patients [2]. In its entirety, COVID-19 has severely injured the global economy and distressed the comforts and wellbeing of our usual lives.

\section{Epidemiology}

The SARS-COV-2 pandemic has undergone 3 main phases in the view of geographical epidemiology. Asia was the first geographical location affected and was the region of origin of the virus, the highest concentrations of clusters and outbreaks were in China followed by South Korea. The first phase took place from late December 2019 to mid-late February. The next region to be affected was the European nations from mid-February onwards. The countries hit worst by the virus in these regions were Italy followed by Spain. The third phase commenced in mid-March when the virus had the USA in its sights hence affecting the American continents [2] (Figure 1 and 2). The epidemiological and demographic characteristics vary according to region.

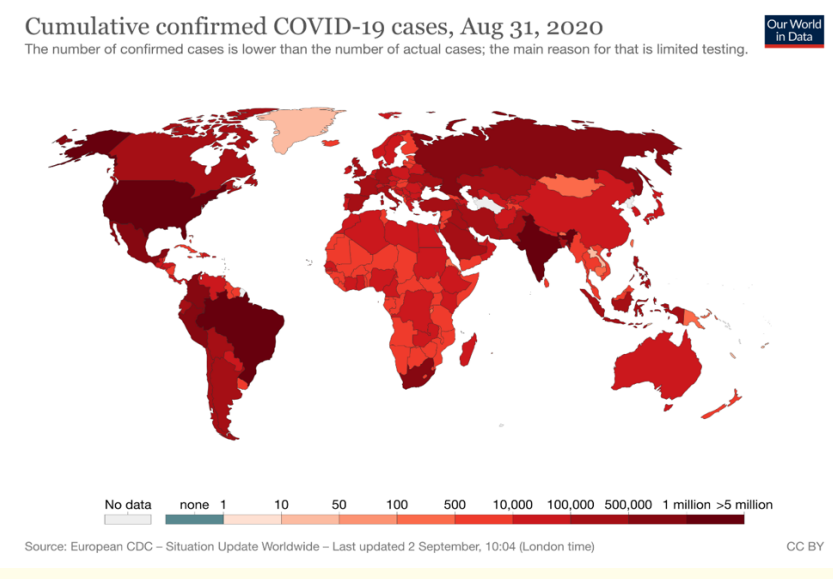

Figure 1: Source [6].

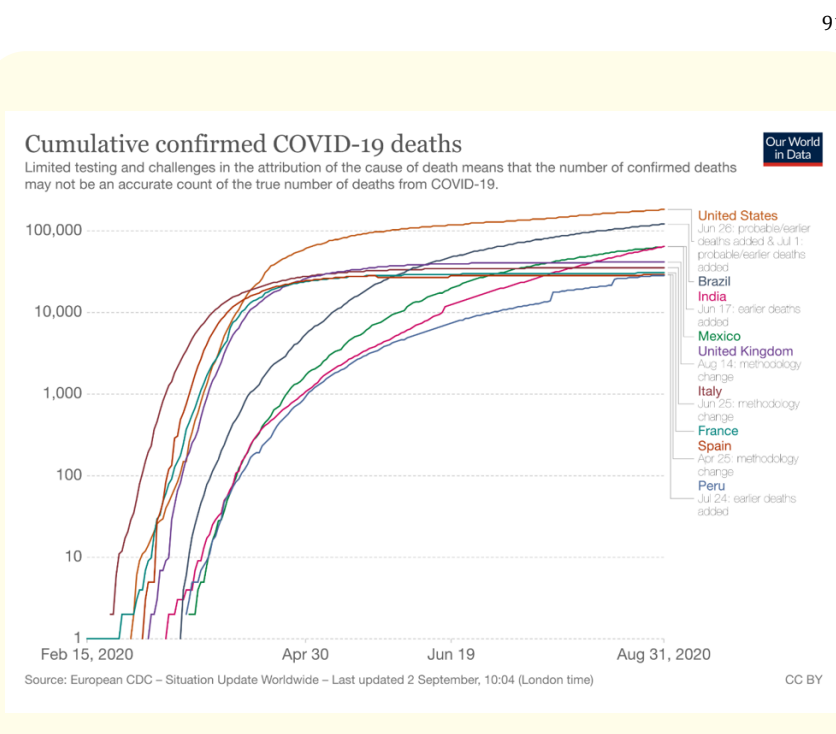

Figure 2: Source [6].

The report of 72314 cases which was released by the Chinese Center for Disease Control and Prevention (CDC), confirmed that most of the cases were aged 30 - 79 years (87\%). Of all the patients $51.4 \%$ were males. $74.7 \%$ of the cases were from the Hubei Province with $85.8 \%$ of them having Wuhan related exposure. The case fatality rate was $14.8 \%$ in patients $>80$ years. $3.8 \%$ of health care personnel were infected. $63 \%$ of which were from Wuhan. The peak of the epidemic curve was seen on January 23 - 26 after which it declined [1,3].

A study in the United Kingdom of 20133 patients showed that the median age of patients was 73 years and $33 \%$ were over the age of 80 . The percentage of male patients was $60 \% .17 \%$ of the patients required high dependency admission or intensive care. $77.5 \%$ had any comorbidity. Increased age, male sex and comorbidities such as chronic cardiac had an associated higher mortality rate in this study. $41 \%$ of the patients were discharged, $26 \%$ died and $34 \%$ were still under therapy at the date of reporting [4].

In the United States, a study of 1,320,488 patients concluded that the cumulative incidence was found to be similar between males (401.1 cases per 100000) and females (406.0 cases per 100000). The median age of infected patients was 48 years. The highest cumulative incidence was found in patients over 80 years old (902 cases per 100000. Hospitalization was 6 times greater in patients with underlying comorbidities (45.4\%) than patients 
without (7.6\%). Deaths were 12 times higher in patients with underlying conditions (19.5\%). Irrespective of underlying comorbidity, death was commonest in patients $>80$ years of age. The 7-day moving average number of new daily cases peaked on the $12^{\text {th }}$ of April and the number of deaths peaked on the $21^{\text {st }}$ of April [5].

In general, the evidence indicates that COVID-19 is more fatal in elderly patients and in those with underlying co-morbidities. Both males and females are almost equally susceptible to acquiring the condition (Figure 3 and 4).

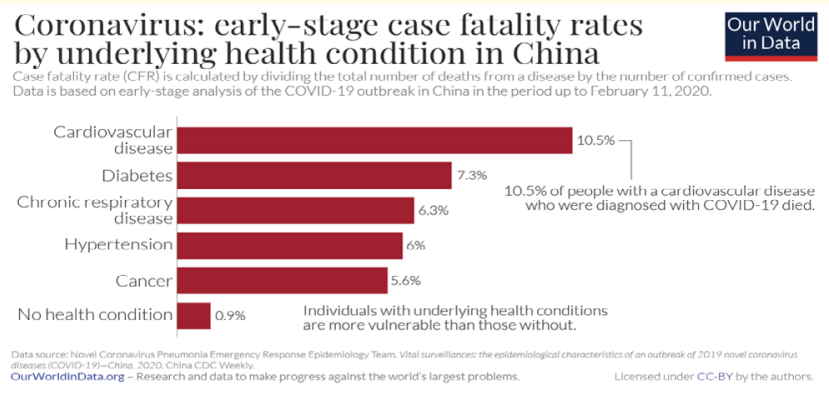

Figure 3: Source [6].

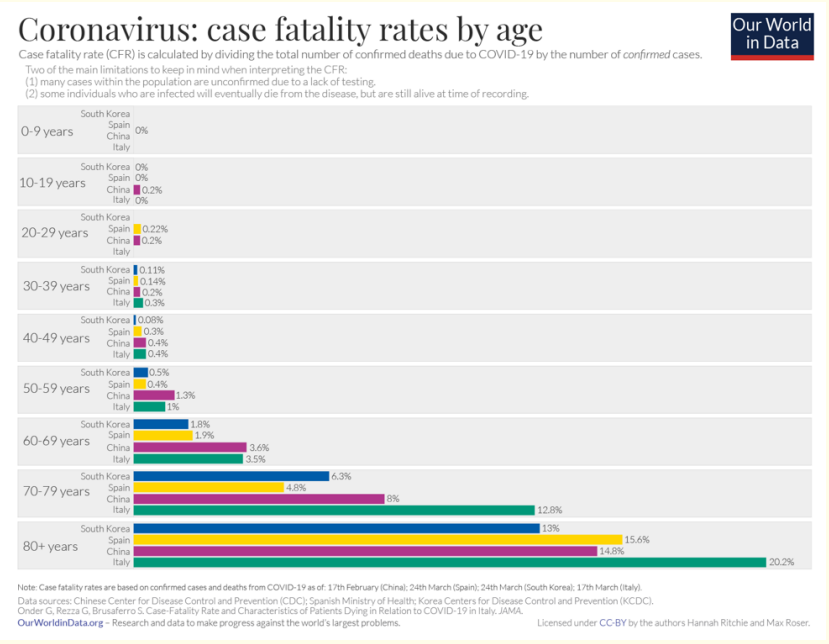

Figure 4: Source [6].

\section{Virology}

Morphology: Coronaviruses are single-stranded positive sense RNA viruses that are classified under the Coronaviridae family. The structure SARS-CoV-2 can be observed in prefixed cells under electron microscopy. When the virus enters the cell, it derives a lipid bilayer from the host cell. The virus embeds surface spike proteins, viral membrane glycoproteins, and envelope proteins on to this acquired lipid bilayer membrane. This combination of structures encapsulate the genetic material; a helical viral RNA with comprising nucleocapsids [7].

Entry and replication mechanism: Human coronaviruses have glycoprotein spikes on their exterior which are responsible for the attachment and permeation of the virus into a host cell. The spike protein (S protein) interacts with angiotensin-converting enzyme 2 (ACE2), which is a key receptor on the host cell that allows the entry of the SARS coronavirus. Once the S protein and ACE2 receptor are bound a conformational change of the Spike Protein will cause the envelope of the virus to fuse with the lipid bilayer membrane of the host via endosomal action. The SARS-COV-2 genetic material (RNA) is now present in the host cell. The most important genes to note are the orf1ab (largest gene in SARS-COV-2) and the orf1a which translates in the host cell to the viral replicase polyproteins pp1a and pp1ab which eventually are cleaved by viral proteinases. Polymerase creates viral mRNA which translate to viral proteins such as the spike, envelope, and membrane proteins. These proteins plus the genomic RNA are assembled in Golgi and ER of the host cell and then trafficked via vesicles and released out to infect other cells [8].

Genomic variation: In comparison to the previous human coronavirus, SAR-COV-2 is said to have more than an $80 \%$ similarity in genotype. A coronavirus genome includes a varying number of open reading frames (ORFS). The first ORF usually contains 67\% of the entire genome and codes for 16 non-structural proteins, the other ORFs code for all other accessory and structural proteins. SARS-COV-2 was found to have 14 ORFs that encode 27 proteins. In terms of amino acids, the SARS-COV-2 has a few significant differences between the SARS-COV. A few being the absence of the $8 \mathrm{a}$ accessory protein in SARS-COV-2, the longer length of $8 \mathrm{~b}$ accessory protein in SARS-COV-2, the shorter $3 \mathrm{~b}$ accessory protein in SARS-COV-2. However, the impact these differences have on the functioning, virulence, and pathogenesis of SAR-COV-2 are yet to be studied [9]. Other studies also prove that the spike glycoprotein of SARS-COV-2 is a combination of bat SARS-CoV and an unidentified Beta-CoV. Some studies show mutations in the spike protein 
of SARS-CoV-2 have a significantly greater binding affinity for the ACE2 host receptor [8].

Primary reservoirs and intermediate reservoirs: In 2010, studies confirmed that the Rhinolophus sinicus (Chinese rufous horseshoe bat) was the natural and primary host of SARS-CoV. The intermediate host was considered to be the civet cat found in a wildlife market. SARS-CoV-2 and SARS-CoV have about $80 \%$ of their genomic sequence in common. The latest studies also report a $96.2 \%$ commonality between SARS-CoV-2 and a coronavirus identified from Rhinolophus affinis which highly suggests an origin from bats. The intermediate host for SARS-CoV-2 is still under study, the closest research suggests the pangolin due to a $99 \%$ similarity in coronavirus infecting pangolins and the human strain SARS-CoV-2 [10].

Human to human transmission: Multiple studies have concluded that the origin of coronavirus is from an animal species and then transmitted among humans. The most common method being the same that of SARS, inhalation of respiratory droplets, or touching contaminated surfaces. The respiratory droplets are released into the air via coughing and sneezing. There is also recent evidence that the SARS-CoV-2 can be identified in the stools of patients and hence justifies probable fecal-oral transmission as well. Transmission via person-to-person is more evident when there is prolonged exposure to an infected individual, thus exposing the patient to a greater viral load [11].

Incubation period: The SARS-CoV has been found to incubate over 1 - 4 days however there are also reports of it lasting about 10 days. In the case of COVID-19, there is evidence that there is about 3 - 7 days, reaching up to 14 days of incubation [10]. Nevertheless, the topic of the exact incubation period is still up for research as multiple recent publications have proposed varying periods of latency and incubation [12].

\section{Clinical features}

Symptoms: The symptoms most encountered in positive cases of COVID-19 are fever (82.2\%), cough (61.7\%), fatigue (44\%) and dyspnea (41\%). These symptoms are not very specific and are shared among many viral respiratory conditions. Symptoms such as myalgia (22.7\%), vomiting (3.6\%) and diarrhea (8.4\%) are rare in COVID-19 cases and hence may insinuate another focus of infection [13].

\section{Laboratory findings and diagnosis}

The most common laboratory abnormality seen in a patient with COVID-19 is eosinopenia (78.8\%), lymphopenia (68.7\%) and, an elevated aspartate aminotransferase (AST) level (63.4\%). Despite the obvious connection between eosinopenia and COVID-19 it has a low sensitivity and specificity and hence cannot be employed for testing [13]. Another analysis of 1099 patients in mainland China identified lymphocytopenia (83.2\%) and thrombocytopenia $(36.2 \%)$ as the predominant laboratory derangements. Elevated $\mathrm{C}$-reactive protein levels were also noted. Patients with increasing severity of the disease had more distinct lab differences [14].

Diagnosis covid-19 is still a challenging issue, several diagnose covid-19, however methods are being used to the definitive test for SARS-CoV-2 is the real-time reverse transcriptase-polymerase chain reaction (RT-PCR) test. The place of antibody tests in the investigation of present and previous cases of COVID-19 remains unclear and contentious. It may have a complementary role in diagnosis, especially when the RT-PCR test is negative in those who remain suspected to have COVID-19.

The most common ancillary laboratory findings in patients are.

- Lymphopenia.

Thrombocytosis:

- Increased prothrombin time (PT)

- Increased lactate dehydrogenase.

\section{Radiological imaging}

A study evaluated 975 CT scans performed at the point of admission of 1099 patients who tested positive for COVID-19, of which $86.2 \%$ had abnormal findings. The most common abnormalities noted were ground-glass opacities (56.4\%) and bilateral patchy shadowing (51.8\%). Localized patchy shadowing and interstitial abnormalities may also be seen [14]. In the earlier stages, a ground glass opacification is more predominant, in later stages it will be accompanied by consolidation. The opacifications are located close to the periphery in the sub-pleural area. CT scans hold a sensitivity of $84-98 \%$ and a specificity of $25-80.5 \%$. They exhibit better diagnostic potential than Chest-X-rays [13]. Chest X-rays may also show small patchy shadows alongside interstitial abnormalities in the peripheral regions early in the disease process. As the disease progresses, the ground glass opacity, consolidation and pleural effusions may appear [12]. 
Chest radiographand CT Scan are the two most common investigations used all over the world to diagnose Covid-19. Ultrasonogram and nuclear imaging are also done in many diagnostic centres. The absolute indications are as follows:

- Imaging is not indicated in patients with suspected COVID-19 and mild clinical features unless they are at risk for disease progression.

- Imaging is indicated in a patient with COVID-19 and worsening respiratory status.

- In a resource-constrained environment, imaging is indicated for medical triage of patients with suspected COVID-19 who present with moderate-severe clinical features and a high pertest probability of disease [1].

- CXR is insensitive in mild or early COVID-19 infection [17]. However, with respect to the relative value of CXR or CT for detecting the presence of viral pneumonia, the experience is vastly different dependent upon community norms and public health directives. When patients are encouraged to present early in the course of their disease, as was the case in Wuhan, China, CXR has little value. The greater sensitivity of CT for early pneumonic changes is more relevant in the setting of a public health approach that required isolation of all infected patients within an environment where the reliability of COVID-19 testing was limited and turnaround times were long [4]. Alternatively, in New York City where patients were instructed to stay at home until they experienced advanced symptoms, CXR was often abnormal at the time of presentation. Equipment portability with imaging performed within an infected patient's isolation room is another factor that may favour CXR in selected populations, effectively eliminating the risk of COVID-19 transmission along the transport route to a CT scanner and within the room housing a CT scanner, particularly in environments lacking PPE. In hospitalized patients CXR can be useful for assessing disease progression and alternative diagnoses such as lobar pneumonia, suggestive of bacterial superinfection, pneumothorax and pleural effusion [3].

\section{Chest radiograph}

Although less sensitive than chest CT, chest radiography is typically the first-line imaging modality used for patients with suspected COVID-19 For ease of decontamination, use of portable radiography units is preferred.
Chest radiographs may be normal in early/mild disease. In those COVID-19 cases requiring hospitalization, 69\% had an abnormal chest radiograph at the initial time of admission and $80 \%$ had radiographic abnormalities sometime during hospitalization. Findings are most extensive about 10 - 12 days after symptom onset.

The most frequent findings are airspace opacities, whether described as consolidation or, less commonly, the distribution is most often bilateral, peripheral, and lower zone predominant.

Although cardiac manifestations of COVID-19 are well-recognized, there is no published evidence of cardiac disease on chest radiographs.
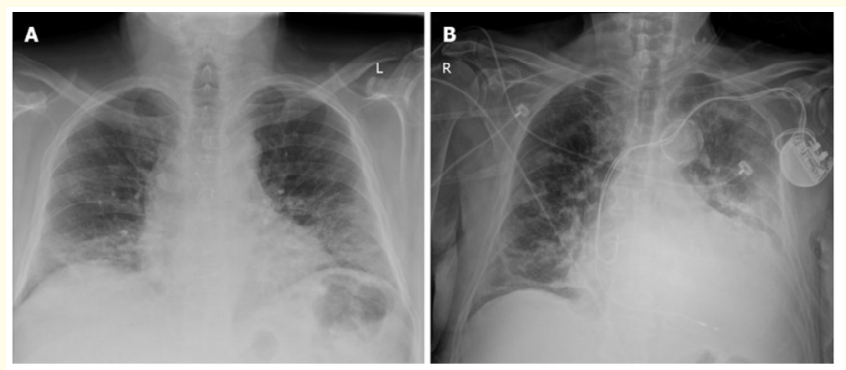

Figure: Anteroposterior chest radiographs in two patients with coronavirus disease 2019 pneumonia from our institution. A: Interstitial infiltrates and ill-defined, patchy, rounded peripheral opacities in bilateral lung fields; B: Interstitial infiltrates with linear and patchy, rounded opacities in bilateral lung fields and heavily calcified aortic arch.

The primary findings on CT in adults have been reported as ground glass opacities bilateral, subpleural, peripheral:

- Air space consolidation.

- Bronchovesicular thickening in the lesion.

- Atypical findings These findings only seen in a small minority of patients should raise concern for superadded bacterial pneumonia or other diagnoses.

- Mediastinal lymphadenopathy.

- $\quad$ Pleural effusion may occur as a complication of COVID-19.

- Multiple tiny pulmonary nodules (unlike many other types of viral pneumonia). 
- Pneumothorax.

- Cavitation.

\section{Complications}

Acute respiratory syndrome: In a study of 201 COVID-19 positive patients, ARDS occurred in 84 patients (41.8\%) and of those 44 died (52.4\%). Risk factors associated with the development and progression of ARDS were older age, neutrophilia, organ/coagulation dysfunction, and D-dimer levels. Patients older than the age of 65 years undergo a more severe degree of ARDS and hence have a higher chance of mortality [15].

Myocardial injury: Theories behind the cause of myocardial injury include: the presence of ACE2 in the myocardial cells enabling viral entry and/or the effect of a cytokine storm causing direct damage. Consequently, acute coronary syndrome, heart failure, myocarditis and arrhythmias may ensue resulting in death. Cardiac markers such as troponin, CK-MB and magnetic resonance imaging can enable diagnosis and predict damage due to COVID-19 [13].

Acute kidney injury: In the progression of COVID-19, increasing severity may cause an elevation in urea and cystatin-C levels suggesting acute kidney injury. The theories of damage are the presence of ACE2 receptors enabling viral entry in the proximal convoluted tubule and the effect of a cytokine storm [13].

Coinfection: It is more common for a COVID-19 patient to be coinfected with bacteria than a virus. Existing studies have reported infection with bacteria causing atypical pneumonia such as Mycoplasma pneumoniae, Chlamydia pneumoniae and Legionella pneumoniae. Encountered co-infecting viruses include respiratory syncytial virus, influenza A and Influenza B [13].

\section{Clinical management of COVID-19}

The first step of management is screening and triage. Screening involves asking questions related to symptomology, exposure, risk factors, etc. Triaging can be done according to a standardized triage tool. Following which the patient must be categorized according to disease severity; mild, moderate, severe and, critical disease. Critical disease encompasses the complications of COVID-19 such as ARDS and Septic Shock. Moderate and Severe is differentiated according to the degree progression of the patients' pneumonia. For confirmation of the condition, a laboratory test must be performed. The recommendation is a reverse transcriptase-polymerase chain reaction testing of an upper respiratory tract sample such as a nasopharyngeal/oropharyngeal swab. If the patient's disease is severe or critical, blood cultures are also recommended [16].

Management of mild COVID-19 can be done at a designated health/community facility or home. The primary goal being isolation to avoid the spread of the condition. The current recommendation is only symptomatic treatment e.g. antipyretics, sufficient nutrition, and hydration. The patient should be well informed of the progression and potential complications of the disease, so that they can seek urgent care when necessary [16].

Management of moderate COVID-19 can be performed at home however is decided on a case-by-case basis. If there is a potential risk of deterioration, hospital admission is required. Close monitoring for disease progression is recommended, such as vital signs, pulse oximetry, and medical early warning scores. Treatment is according to symptoms. Empirical antibiotics can be considered for older people and children less than 5 years. For other groups of patients, it is not recommended unless a bacterial infection is highly suspected [16].

Management of severe COVID-19 must be conducted in a health care facility. It should be adequately equipped with an oxygen delivery mechanism and pulse oximeters. Supplemental oxygen is recommended when there are emergency signs or when the $\mathrm{SPO}_{2}$ $<90 \%$. There should be close monitoring for signs of respiratory failure and shock. Hematological and biochemical testing, imaging and ECG must be performed on admission and as clinically indicated. Timely supportive therapy in the case of complications such as ARDS, DIC, and cardiac injury is key. Fluid management should be performed cautiously [16].

Management of critical COVID-19 with ARDS differs according to the severity of the ARDS. In mild ARDS the recommendation is a trial of high-flow nasal oxygen or non-invasive ventilation with continuous positive airway pressure or bi-level positive airway pressure. If the ARDS is of higher severity, hypoxemic respiratory failure must be immediately identified and endotracheal intubation must be performed to provide mechanical ventilation [16].

Management of critical COVID-19 with Septic Shock involves early recognition and therapy to maintain an adequate mean arterial pressure. A rapid bolus of crystalloid fluid followed by vaso- 
pressors such are norepinephrine and epinephrine can be administered if the shock persists [16].

The WHO recommends against the use of antiviral therapy, corticosteroids, immunomodulators, plasma therapy, and other adjunctive therapy as prophylaxis or treatment.

If the patient recovers, adequate psychosocial support, and tailored rehabilitation programs should be provided. In the case of continuous deterioration, the patients advance care plan should be discussed and the necessity for palliative care must be explored [16].

Hydroxychloroquine, chloroquine, azithromycin and anti-virals as therapy for COVID-19

A study conducted among 821 asymptomatic patients across the US and Canada who were exposed to someone with confirmed Covid-19 concluded that COVID-19 was not prevented by hydroxychloroquine when implemented as post-exposure prophylaxis within 4 days of high to moderate exposure. Hydroxychloroquine was confirmed to have side effects of nausea, loose stools, and abdominal discomfort [17].

A small study involving 20 treated patients in France reveals that hydroxychloroquine produces a significant reduction in viral load. With the addition of Azithromycin, the effect was further potentiated. According to the report, $70 \%$ of patients treated with hydroxychloroquine were virologically cured on day 6 post-inclusion, whereas the control group showed just $12.5 \%$ [18].

A meta-analysis published in the Annals of Internal Medicine, taking into account 4 randomized control trials, 10 cohort studies 9 case series concluded that the advantages and disadvantages of using hydroxychloroquine or chloroquine in the therapy of COVID-19 are very poor and conflicting. The analysis reports ECG abnormalities of QTc intervals of $500 \mathrm{~ms}$ or more in patients receiving hydroxychloroquine. Similar statements were made with Chloroquine. An observational study reported minor fever resolution and increased viral clearance in patients prescribed chloroquine than those not prescribed chloroquine [19].

A since retracted paper from The Lancet with a study sample of 96032 patients was unable to confirm the benefit of hydroxychloroquine or chloroquine, used by itself, or in combination with a macrolide. Nevertheless, it was also noted that there were a lessened in-hospital survival and greater frequency of ventricular arrhythmias [20].

A statement released by the RECOVERY trial on the $5^{\text {th }}$ of June, which randomized 1542 patients to hydroxychloroquine has concluded that there is no beneficial effect in terms of hospital stay or mortality rates.

A cohort study in Boston with 90 participants of which 53 received azithromycin in combination revealed that the patients were at a higher risk of QTc prolongation. Other reported adverse effects were hypoglycemia, intractable nausea, and a single case of torsade's de pointes [21].

In the view of antiviral therapy, when administered early a triple combination of interferon beta $1 \mathrm{~b}$, lopinavir-ritonavir and ribavirin was safe and shortened the duration of hospital stay, concluded a study in Hong Kong [22]. However, other antivirals such as remdesivir showed no evidence of clinical benefit but MAY have beneficial effects if prescribed earlier in the course of the disease which requires verification in a bigger study [23].

A prospective, randomized, controlled, open labeled multicenter trial testing the use of favipiravir an oral antiviral concluded that there was no significant improvement in the clinical recovery rate by Day 7 of illness of COVID-19. However, it significantly improved the time taken to attain relief from cough and fever with mild and minimal adverse effects [24]. Favipiravir has also been found to be associated with a faster viral clearance and a significantly better improvement rate in the chest CT scans. The control arm of this study administered lopinavir/ritonavir to the affected patients, these patients were found to have greater adverse effects in comparison to those administered favipiravir [25].

Overall, the evidence available regarding the use of hydroxychloroquine, azithromycin, and antivirals as adjunctive or prophylactic therapy for COVID-19 is conflicting and insufficient. The WHO also recommends against the use of it [16] and hence responsible clinical action would be to await more decisive evidence on the topic.

\section{Vaccinations}

As of the $20^{\text {th }}$ of August 2020, there are 6 candidate vaccines in the $3^{\text {rd }}$ phase of clinical trials. The goal of a phase 3 trial is to test the effectiveness of a particular intervention over a large group of 
human subjects over a long duration of time. The size and duration of phase 3 trials usually make them very expensive to conduct.

The vaccine developed by Moderna uses mRNA-1273 which enables the production of the spike protein in patient cells without beings infected by the virus. The Phase 3 study began on the $27^{\text {th }}$ of July. The vaccine requires 2 doses given 28 days apart. All 45 participants in the Phase one trial demonstrated serum neutralizing activity after the second dose [26,27].

A partnership between the University of Oxford and AstraZeneca has developed a vaccine from a weakened version of the adenovirus extracted from chimpanzees. AstraZeneca is to supply 400 million doses across Europe, delivered by the end of 2020. When vaccinated the surface spike protein will be developed which will enable the immune system to provide an adequate secondary response when infected with SARS-CoV-2. The recombinant adenovirus vector (ChAdOx1) has been vaccinated to 320 people as of $13^{\text {th }}$ June and is shown to be well tolerated [28].

Sinovac is in phase 3 of testing an inactivated vaccine against SARS-CoV-2. The vaccination plan is a 0,14 schedule. Results of phase 1 and 2 showed no serious adverse effect among the 743 volunteers and more than $90 \%$ seroconversion was seen after the second dose of the vaccine [29].

\section{Prevention and policy responses}

Interim guidance by the World Health Organization in late May elaborated public health and social measures that can be employed to stop chains of transmission, prevent outbreaks and limit further spread of SARS-CoV-2 [30].

Personal measures include hand hygiene, physical distancing, use of masks, and respiratory etiquette. The goal of these measures is to limit spread between individuals, provide adequate protection for the vulnerable and reduce contamination of surfaces [30].

Physical and social distancing measures aim to limit transmission in public spaces. Measures recommended are cancellation of mass groups or congregations, avoiding crowded settings such as public transport and restaurants, working from home, staying at home. The recommended physical distancing is at least one meter apart [30].

Movement measures are to prevent the migration of the virus from one geographical area to another. Measures recommended are limiting international and local movement, arranging orderly travel to avoid congestion, and providing updated guidance on traveling [30].

Special protection measures are primarily to support and protect populations at a higher risk of contracting the virus such as older people, closed settings (detention centers), frontline workers, etc. Some measures include limitation of visitors, separation from others, ensuring the availability of PPE, supporting telemedicine, and remote health services.

A recent study into the effectiveness of disease prevention policies and methods has further emphasized the importance of how a combination of strategies such as isolation, tracing, quarantine reduce transmission better [31].

\section{Conclusion}

COVID-19 is caused by SARS CoV-2 with a fatality rate lying approximately between $0.5 \%$ to $10 \%$ worldwide [32] (Figure 5). Patients with underlying systemic illness and the elderly have the highest risk of contracting the disease and having a fatal reaction to it. Human to human transmission via aerosolized respiratory droplets is the most common form of transmission. The majority of all patients $(80 \%)$ are asymptomatic or have a milder presentation. There is insufficient evidence to suggest transmission from asymptomatic patients [33,34]. Early identification, isolation, and symptomatic management of infected patients is the minimum recommended practice worldwide. The use of adjunctive therapy such as hydroxychloroquine and antivirals are not recommended. 6 vaccines are currently entering into the final phase 3 of their clinical trials. Prevention strategies are implemented worldwide to halt the spread of the virus. Masks, movement restrictions, physical distancing, and quarantine measures are a few among the effective. We have to live with COVID-19 pandemic for several years with all precautions of infection control use of mask, frequent hand wash with soap and sanitizers and safe distance despite COVID vaccination we have it will take minimum time of one to two years for development of 80 percent herd immunity which will result in dropping of new cases.

\section{Acknowledgements}

I am highly thankful to Professor Dr Mohd Raili Suhaili, Dean Faculty of Medicine, Segi University, Sibu sub-campus, hospital Sibu, Sarawak state Malaysia, and Mrs Margareth Wong, Clinical 
Total confirmed COVID-19 deaths vs. cases, Aug 31, 2020 The number of confirmed cases is lower than the number of total cases. The main reason for this is limited testing.
The grey lines show the corresponding case fatality rates, CFA (the ratio between confirmed deaths and confirmed
cases).

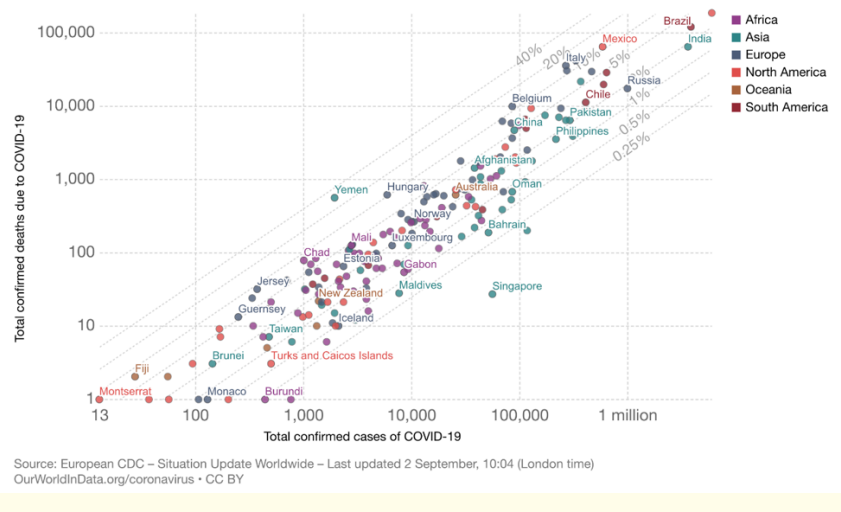

Figure 5: Source [6].

Community Research Manager, faculty of medicine, clinical campus, hospital Sibu, Sibu city 96000 (Sarawak state) for their valuable guidanceconstant encouragement and inspiration.

\section{Bibliography}

1. $\mathrm{Wu} \mathrm{Z}$ and McGoogan JM. "Characteristics of and Important Lessons From the Coronavirus Disease 2019 (COVID-19) Outbreak in China: Summary of a Report of 72314 Cases From the Chinese Center for Disease Control and Prevention". The Journal of the American Medical Association 323.13 (2020): 1239-1242.

2. Pericàs JM., et al. "COVID-19: from epidemiology to treatment". European Heart Journal 41.22 (2020): 2092-20112.

3. The Novel Coronavirus Pneumonia Emergency Response Epidemiology T. The Epidemiological Characteristics of an Outbreak of 2019 Novel Coronavirus Diseases (COVID-19) - China, 2020". China CDC Weekly 2.8 (2020): 113-122.

4. Docherty AB., et al. "Features of 20133 UK patients in hospital with covid-19 using the ISARIC WHO Clinical Characterisation Protocol: prospective observational cohort study". BMJ 369 (2020): m1985.

5. Stokes E., et al. "Coronavirus Disease 2019 Case Surveillance United States". MMWR Morbidity and Mortality Weekly Report (2020): 69.
6. Roser M., et al. "Coronavirus Pandemic (COVID-19)". Our World in Data (2020).

7. Kumar S., et al. "Morphology, Genome Organization, Replication, and Pathogenesis of Severe Acute Respiratory Syndrome Coronavirus 2 (SARS-CoV-2)". In: Saxena SK, editor. Coronavirus Disease 2019 (COVID-19): Epidemiology, Pathogenesis, Diagnosis, and Therapeutics. Singapore: Springer Singapore (2020): 23-31.

8. Shereen MA., et al. "COVID-19 infection: Origin, transmission, and characteristics of human coronaviruses". Journal of Advanced Research 24 (2020): 91-98.

9. Wu A., et al. "Genome Composition and Divergence of the Novel Coronavirus (2019-nCoV) Originating in China". Cell Host and Microbe 27.3 (2020): 325-328.

10. Xu J., et al. "Systematic Comparison of Two Animal-to-Human Transmitted Human Coronaviruses: SARS-CoV-2 and SARSCoV". Viruses 12.2 (2020).

11. Yadav T and Saxena SK. "Transmission Cycle of SARS-CoV and SARS-CoV-2". In: Saxena SK, editor. Coronavirus Disease 2019 (COVID-19): Epidemiology, Pathogenesis, Diagnosis, and Therapeutics. Singapore: Springer Singapore (2020): 33-42.

12. Wu D., et al. "The SARS-CoV-2 outbreak: What we know". International Journal of Infectious Diseases 94 (2020): 44-48.

13. Siordia JA. "Epidemiology and clinical features of COVID-19: A review of current literature". Journal of Clinical Virology 127 (2020): 104357.

14. Guan W-J., et al. "Clinical Characteristics of Coronavirus Disease 2019 in China". New England Journal of Medicine 382.18 (2020): 1708-1720.

15. Wu C., et al. "Risk Factors Associated With Acute Respiratory Distress Syndrome and Death in Patients With Coronavirus Disease 2019 Pneumonia in Wuhan, China". JAMA Internal Medicine (2020).

16. Clinical management of COVID-19: Interim Guidance, 27 May 2020: World Health Organization (2020).

17. Boulware DR., et al. "A Randomized Trial of Hydroxychloroquine as Postexposure Prophylaxis for Covid-19". New Eng- 
land Journal of Medicine (2020).

18. Gautret P., et al. "Hydroxychloroquine and azithromycin as a treatment of COVID-19: results of an open-label non-randomized clinical trial". International Journal of Antimicrobial Agents (2020): 105949.

19. Hernandez AV., et al. "Hydroxychloroquine or Chloroquine for Treatment or Prophylaxis of COVID-19: A Living Systematic Review". Annals of Internal Medicine (2020).

20. Mehra MR., et al. "RETRACTED: Hydroxychloroquine or chloroquine with or without a macrolide for treatment of COVID-19: a multinational registry analysis". The Lancet (2020).

21. Mercuro NJ., et al. "Risk of QT Interval Prolongation Associated With Use of Hydroxychloroquine With or Without Concomitant Azithromycin Among Hospitalized Patients Testing Positive for Coronavirus Disease 2019 (COVID-19)". JAMA Cardiology (2020).

22. Hung IF-N., et al. "Triple combination of interferon beta-1b, lopinavir-ritonavir, and ribavirin in the treatment of patients admitted to hospital with COVID-19: an open-label, randomised, phase 2 trial". The Lancet 395.10238 (2020): 16951704.

23. Wang Y., et al. "Remdesivir in adults with severe COVID-19: a randomised, double-blind, placebo-controlled, multicentre trial". The Lancet 395.10236 (2020): 1569-1578.

24. Chen C., et al. "Favipiravir versus Arbidol for COVID-19: A Randomized Clinical Trial”. Med Rxiv (2020).

25. Cai Q., et al. "Experimental Treatment with Favipiravir for COVID-19: An Open-Label Control Study”. Engineering (2020).

26. Moderna's Work on a COVID-19 Vaccine Candidate (2020).

27. Jackson LA., et al. "An mRNA Vaccine against SARS-CoV-2 - Preliminary Report". New England Journal of Medicine (2020).

28. Astra Zeneca to supply Europe with up to 400 million doses of Oxford University's vaccine at no profit: @astrazeneca (2020).

29. Sinovac COVID-19 Vaccine Collaboration with Butantan Receives Approval from Brazilian Regulator for Phase III Trial (2020).
30. Overview of Public Health and Social Measures in the context of COVID-19. World Health Organization (2020).

31. Kucharski AJ., et al. "Effectiveness of isolation, testing, contact tracing, and physical distancing on reducing transmission of SARS-CoV-2 in different settings: a mathematical modelling study". The Lancet Infectious Diseases (2020).

32. Mortality Analyses Johns Hopkins University and Medicine (2020).

33. Coronavirus disease 2019 (COVID-19). Situation Report - 73: World Health Organization (2020).

34. Coronavirus disease 2019 (COVID-19). Situation Report - 46. World Health Organization (2020).

35. Heshui Shi., et al. "Radiological findings from 81 patients with COVID-19 pneumonia in Wuhan, China: a descriptive study". Lancet 12.8 (2020): 142-155.

36. Audrey E Kaufman., et al. "Review of radiographic findings in COVID-19" (2020).

- Prompt Acknowledgement after receiving the article

- Thorough Double blinded peer review

- Rapid Publication

- Issue of Publication Certificate

- High visibility of your Published work Assets from publication with us Website: www.actascientific.com/

Submit Article: www.actascientific.com/submission.php Email us: editor@actascientific.com

Contact us: +919182824667 